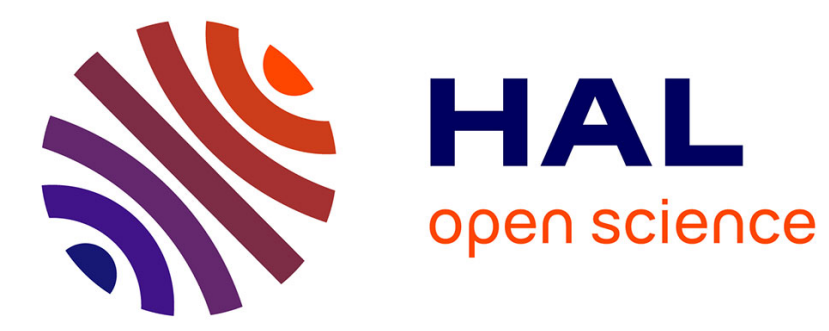

\title{
Ultrafast dynamical response of the lower exciton-polariton branch in CdZnTe
}

\author{
J. Lohrenz, S. Melzer, C. Ruppert, I. Akimov, H. Mariette, M. Reichelt, A. \\ Trautmann, T. Meier, M. Betz
}

\section{- To cite this version:}

J. Lohrenz, S. Melzer, C. Ruppert, I. Akimov, H. Mariette, et al.. Ultrafast dynamical response of the lower exciton-polariton branch in CdZnTe. Physical Review B, 2016, 93 (7), pp.075201. 10.1103/PhysRevB.93.075201 . hal-01949943

\section{HAL Id: hal-01949943 \\ https://hal.science/hal-01949943}

Submitted on 25 Feb 2021

HAL is a multi-disciplinary open access archive for the deposit and dissemination of scientific research documents, whether they are published or not. The documents may come from teaching and research institutions in France or abroad, or from public or private research centers.
L'archive ouverte pluridisciplinaire HAL, est destinée au dépôt et à la diffusion de documents scientifiques de niveau recherche, publiés ou non, émanant des établissements d'enseignement et de recherche français ou étrangers, des laboratoires publics ou privés. 


\title{
Ultrafast dynamical response of the lower exciton-polariton branch in CdZnTe
}

\author{
J. Lohrenz, ${ }^{1}$ S. Melzer, ${ }^{1}$ C. Ruppert, ${ }^{1}$ I. A. Akimov, ${ }^{1}$ H. Mariette,${ }^{2}$ M. Reichelt, ${ }^{3}$ A. Trautmann, ${ }^{3}$ T. Meier, ${ }^{3}$ and M. Betz ${ }^{1}$ \\ ${ }^{1}$ Experimentelle Physik 2, TU Dortmund, Otto-Hahn-Strasse 4, 44221 Dortmund, Germany \\ ${ }^{2}$ CEA-CNRS Group Nanophysique et Semiconducteurs, Institut Neel, Université Grenoble-Alpes, 25 Avenue des Martyrs, \\ 38042 Grenoble, France \\ ${ }^{3}$ Department Physik \& CeOPP, Universität Paderborn, Warburger Strasse 100, D-33098 Paderborn, Germany
}

(Received 13 December 2015; revised manuscript received 13 January 2016; published 2 February 2016)

\begin{abstract}
We investigate the transient optical response in high-quality $\mathrm{Cd}_{0.88} \mathrm{Zn}_{0.12}$ Te crystals in the regime of slow light propagation on the lower exciton-polariton branch. Femtosecond photoexcitation leads to very substantial transmission changes in a $\sim 10-\mathrm{meV}$ broad spectral range within the transparency window of the unexcited semiconductor. These nonlinear optical signatures decay on picosecond time scales governed by carrier thermalization and recombination. The temporal and spectral dependence indicate the dynamical optical response as arising from excitation-induced dephasing and perturbed free induction decay. Model simulations for the optical response taking into account the actual exciton-polariton dispersion and excitation-induced dephasing of a nonlinearly driven two-level system support this interpretation.
\end{abstract}

DOI: 10.1103/PhysRevB.93.075201

\section{INTRODUCTION}

For photon energies close to the fundamental exciton resonance of a high-quality bulk semiconductor, light propagation is substantially slowed down when compared to a regular dielectric. This phenomenon originates from the emergence of a mixed state between the incident electromagnetic radiation and the induced polarization wave, i.e., the formation of exciton polaritons [1,2]. In particular, for light propagating on the lower polariton branch, the group velocity can become several orders of magnitude smaller than the speed of light in vacuum [3-5] and, ultimately, reach values as low as typical speeds of sound [6]. This slow propagation leads to substantial time delays of up to $1 \mathrm{~ns}$, even in submillimeter-thick crystals [7-9]. It has been evidenced by time-of-flight measurements in various semiconductor materials. Less attention has been paid to the dynamical control over the exciton-polariton propagation, e.g., by the photoinjection of charge carriers into the semiconductor. Transient optical studies have so far mainly focused on pump-probe experiments involving photon energies right at [10] or somewhat above the exciton resonance [11]. In this regime, the excitonic admixture to the exciton polaritons is large such that these quasiparticles are strongly susceptible to excitation-induced dephasing. Correspondingly, the optical nonlinearity in close vicinity to the exciton resonance is substantial, and pronounced modifications of the absorption edge of a semiconductor are seen upon photoexcitation. However, also for photon energies slightly below the onset of the optical absorption, the excitonic contribution to the lower polariton branch is substantial. In addition, the negligible residual absorption permits the use of crystals thick compared to the typical penetration depth related to the interband absorption. In combination with the reduced group velocity, therefore, relatively long interaction times are feasible which effectively enhance the impact of any excitation on light propagating on the lower polariton branch.

In this article we investigate the ultrafast optical response of the lower polariton branch in high-quality $\mathrm{Cd}_{0.88} \mathrm{Zn}_{0.12} \mathrm{Te}$ crystals of $\sim 200-\mu \mathrm{m}$ thickness. Most strikingly, photoexcitation leads to transmission changes of several tens of percent in a $\sim 10-\mathrm{meV}$ broad spectral range within the transparency window of the unexcited semiconductor. These nonlinear optical signatures decay on picosecond time scales governed by carrier relaxation and recombination. By analyzing the temporal and spectral dependence of the transient optical signatures, we identify the response as arising from excitationinduced dephasing related to scattering with charge carriers, as well as from the perturbed free induction decay of the exciton resonance. Model simulations for the optical response of a two-level system in the $\chi^{(3)}$ limit including polariton propagation effects corroborate this interpretation.

\section{EXPERIMENTAL SETUP AND SAMPLE}

The experiments rely on a nondegenerate femtosecond pump-probe configuration. The optical source is a $230-\mathrm{kHz}$ Ti:sapphire regenerative amplifier delivering $\sim 40$-fs pulses centered at $1.55-\mathrm{eV}$ photon energy. A fraction of this pulse train serves as a pump pulse. The remainder is fed into an optical parametric amplifier (OPA) to generate near-infrared pulses of $\sim 60$-fs duration and $0.8-\mathrm{eV}$ central photon energy. The second harmonic of this OPA pulse is utilized as a broadband probe pulse. Its transmission is analyzed either in a spectrally integrated fashion or spectrally dispersed in a monochromator of $\sim 1-\mathrm{meV}$ resolution. The analysis of transient transmission changes relies on standard lock-in analysis referenced to a $\sim 2.3-\mathrm{kHz}$ modulation of the excitation beam. The investigated sample is a 208- $\mu$ m-thick, (100)-oriented $\mathrm{Cd}_{0.88} \mathrm{Zn}_{0.12}$ Te crystal with a background $p$-type doping on the order of $10^{15} \mathrm{~cm}^{-3}$. It is kept in a flow cryostat at $T \sim 8 \mathrm{~K}$. We first want to relate the pump and probe photon energies of the experiment to the upper and lower exciton-polariton resonances of $\mathrm{Cd}_{0.88} \mathrm{Zn}_{0.12} \mathrm{Te}$, cf. Fig. 1(a). The energetic positions of the resonances are extracted from the linear transmission spectrum of the sample and are consistent with previous work analyzing time-of-flight measurements on a $745-\mu \mathrm{m}$-thick specimen of the same wafer [8]. The pump pulse is centered at around $1.550 \mathrm{eV}$ and is, thus, clearly below the excitonic resonance energy of $1.6638 \mathrm{eV}$ at cryogenic temperatures. Hence, excitation of the sample 

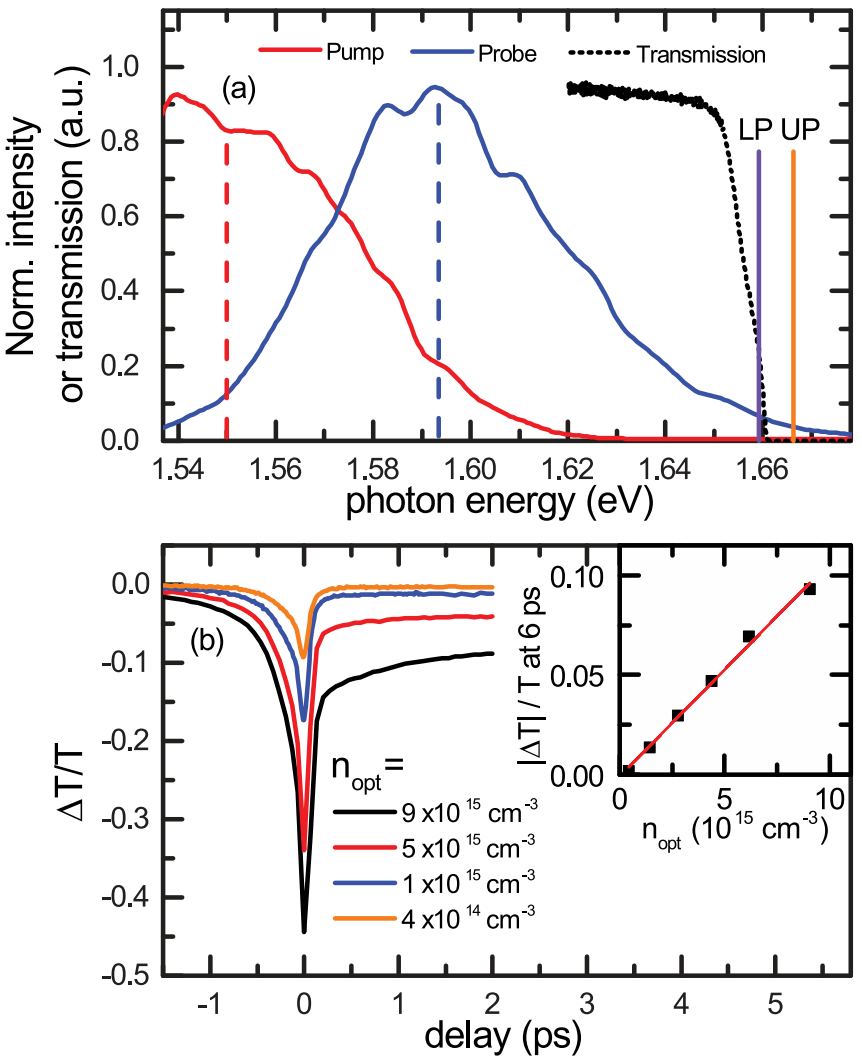

FIG. 1. (a) Normalized spectra of the pump (red) and probe (blue) pulses. Dashed line: linear transmission spectrum of the $208-\mu \mathrm{m}-$ thick $\mathrm{Cd}_{0.88} \mathrm{Zn}_{0.12}$ Te sample. The vertical lines indicate the location of the upper (UP, 1.6637-eV) and lower (LP, 1.657-eV) polariton resonances. (b) Normalized differential transmission transients for various excitation densities. The inset shows the signal detected at $\tau_{D}=6 \mathrm{ps}$ as a function of the optical excitation intensity $n_{\mathrm{opt}}$.

occurs exclusively via two-photon absorption of the ultrashort pump. The choice of this excitation scheme also ensures a rather uniform excitation throughout the entire sample depth. The probe pulse is centered at $1.594 \mathrm{eV}$ and covers the entire spectral region from $\sim 1.54 \mathrm{eV}$ to beyond the lower (LP) and upper (UP) polariton resonances, located at $1.6637 \mathrm{eV}$ and $1.657 \mathrm{eV}$, respectively. However, as the transmission is negligible for photon energies $>1.66 \mathrm{eV}$ [cf. dotted line in Fig. 1(a)], no information about the optical transmission can be recorded beyond this photon energy.

\section{EXPERIMENTAL RESULTS}

We now turn to the discussion of spectrally integrated relative transmission changes after photoexcitation. Figure 1(b) compares four transients recorded for pump irradiances ranging from $30 \mu \mathrm{J} / \mathrm{cm}^{2}$ to $190 \mu \mathrm{J} / \mathrm{cm}^{2}$. From the pump transmission we also extract a two-photon absorption coefficient of $\beta \approx 22 \mathrm{~cm} \times \mathrm{GW}^{-1}$ comparable with the value obtained in CdTe at a photon energy of $1.17 \mathrm{eV}$ [12]. As a result, the excitation levels in Fig. 1(b) correspond to average carrier densities ranging from $4 \times 10^{14} \mathrm{~cm}^{-3}$ to $9 \times 10^{15} \mathrm{~cm}^{-3}$. Most strikingly, a substantial suppression of the optical transmission is found even for moderate carrier densities. It is most

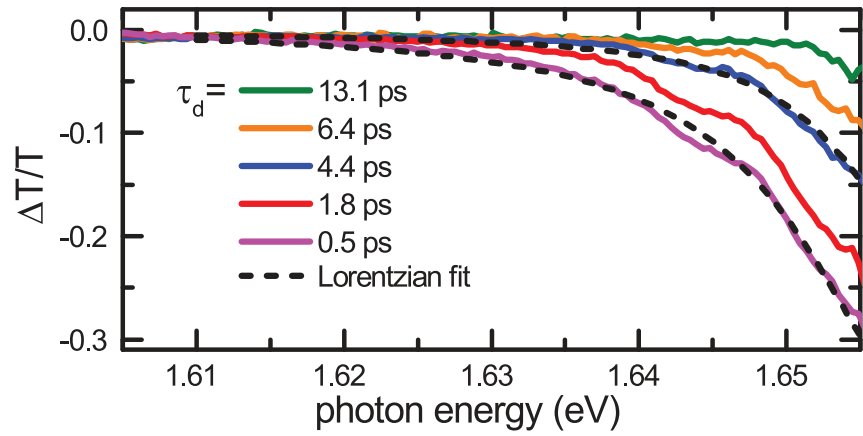

FIG. 2. Spectrally resolved differential transmission for excitation densities of $n=1 \times 10^{15} \mathrm{~cm}^{-3}\left(55 \mu \mathrm{J} / \mathrm{cm}^{2}\right.$ irradiance $)$. The spectra are taken for various positive pump-probe delay times $\tau_{d}>0$. The dashed lines are Lorentzian fits to the data.

pronounced right at the nominal temporal overlap $\left(\tau_{d}=0\right)$ of pump and probe pulses where also two-photon absorptions involving one pump and one probe photon strongly enhance the nonlinear optical response. Note that a delay time $\tau_{d}=0$ corresponds to pump-probe overlap at the front surface of the semiconductor. Remarkably, spectrally averaged transmission changes of up to $\sim 10 \%$ are seen at positive delay times. As visualized in the inset of Fig. 1(b), the transient signals scale linearly with the excitation density $n_{\text {opt }}$ pointing to a $\chi^{(3)}$ regime of this nonlinear optical response. Overall, the transient response decays on time scales of hundreds of picoseconds related to carrier relaxation and recombination (data not shown). The transmission changes also extend to slightly negative delay times of up to $\sim 2 \mathrm{ps}$. This finding is directly related to the slow light propagation for the spectral components of the probe close to the excitonic resonance. In particular, the group velocity on the lower polariton branch is smaller than those of the pump such that the excitation front interacts with the slower components, even at nominally negative delay times.

For a more detailed discussion of the different signal components, we now turn towards differential transmission spectra recorded for a fixed excitation density. Figures 2 and 3 show such spectra for a moderate excitation density of

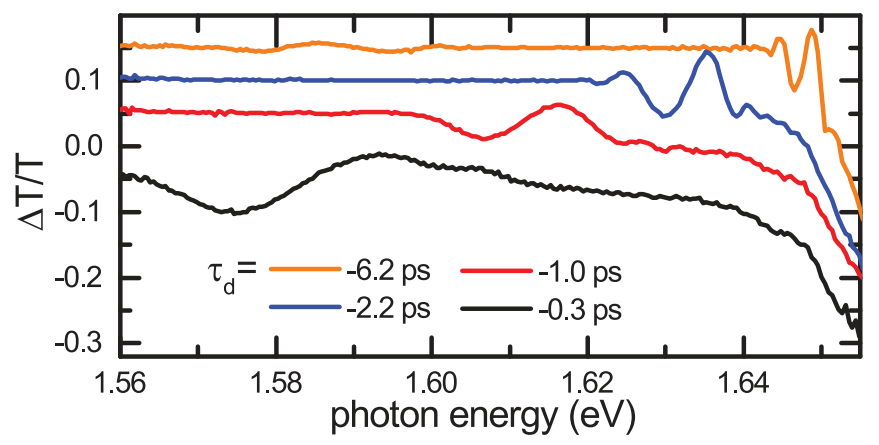

FIG. 3. Spectrally resolved differential transmission for excitation densities of $n=1 \times 10^{15} \mathrm{~cm}^{-3}\left(55 \mu \mathrm{J} / \mathrm{cm}^{2}\right.$ irradiance). The spectra are taken for nominally negative delay times $\tau_{d}<0$ (pump precedes probe pulse at the front surface of the sample). The curves for different delay times shifted by 0.05 each for clarity. Note the different abscissa compared to Fig. 2. 
$1 \times 10^{15} \mathrm{~cm}^{-3}$ (corresponding to an irradiance of $50 \mu \mathrm{J} / \mathrm{cm}^{2}$ ) and a lattice temperature of $T \approx 8 \mathrm{~K}$. We first discuss the experimental results for positive delay times in Fig. 2. Shortly after photoexcitation, the optical transmission in the vicinity of the lower polariton resonance is massively attenuated. This finding can be intuitively attributed to excitation-induced dephasing. In particular, the resonance gets broadened and extends its absorption tail into the transparency region of the unexcited semiconductor. The black dashed lines are Lorentzian fits to the data with a central photon energy at the lower polariton (LP) resonance. (Note that signals related to the upper polariton resonance are not expected because of the thick sample.) They agree rather well with the experimental finding. As an example, for the fit function at $\tau_{D}=0.5 \mathrm{ps}$ we use a width parameter of $w=19 \mathrm{meV}$ for the Lorentzian fit. The remaining discrepancies between the experiment and fit curve are likely related to backreflections of the pump which interact with the probe at effectively negative delay times, which in turn leads to a small signal contribution with more complex shape discussed below. As is also evident from Fig. 2, these induced absorptions markedly decay on time scales as short as several picoseconds. Apparently, the thermalization of the highly energetic photogenerated carriers leads to a reduction of excitation-induced dephasing of the exciton polaritons. For longer delay times, a further reduction of the transient absorption signatures is seen. It originates from the carrier recombination on subnanosecond time scales. In Fig. 3 the corresponding transient spectra for nominally negative delay times $t_{D}$ are depicted. (The different curves are shifted vertically for clarity.) These signals feature spectral oscillations with a period which decreases with increasing absolute value of the delay time. This finding is indicative of a perturbed free induction decay of an exciton polarization and has previously been observed, e.g., in GaAs [13,14]. The temporal extent to which such oscillations are visible points to the coherence time of the excitonic polarization. For the present temperature and excitation density we find $T_{2} \sim 10 \mathrm{ps}$. We note that this time scale is reasonable, as $\hbar / T_{2}=66 \mu \mathrm{eV}$ is consistent with the value of $\sim 10 \mu \mathrm{eV}$ estimated from transmission spectra at lower temperatures $(T=2 \mathrm{~K})$ [15].

\section{SIMULATION RESULTS}

We now attempt to more accurately explain the underlying physics of the pump-probe experiment. We use a $\chi^{(3)}$ perturbation expansion [16] of the incoming fields, taking into account excitation-induced dephasing [17] as the dominant optical nonlinearity. In a simplified model the perturbative expansion of a two-level system leads to hierarchically structured iterative equations:

$$
\begin{aligned}
\frac{\partial}{\partial t} p_{\text {probe }}^{(1)}(t) & =\left(-i \omega_{R}-\gamma\right) p_{\text {probe }}^{(1)}(t)+i \frac{\mu}{\hbar} E_{\text {probe }}(t) \\
\frac{\partial}{\partial t} \delta p^{(3)}(t) & =\left(-i \omega_{R}-\gamma\right) \delta p^{(3)}(t)-\alpha n_{\text {pump }}^{(2)}(t) p_{\text {probe }}^{(1)}(t) .
\end{aligned}
$$

Here, $\omega_{R}$ is the transition frequency of the two-level system, $\gamma$ is the dephasing rate $1 / T_{2}$, and $\mu$ the dipole matrix element. The linear polarization $p_{\text {probe }}^{(1)}$ is driven by the probe pulse. It exists for several picoseconds as the probe pulse

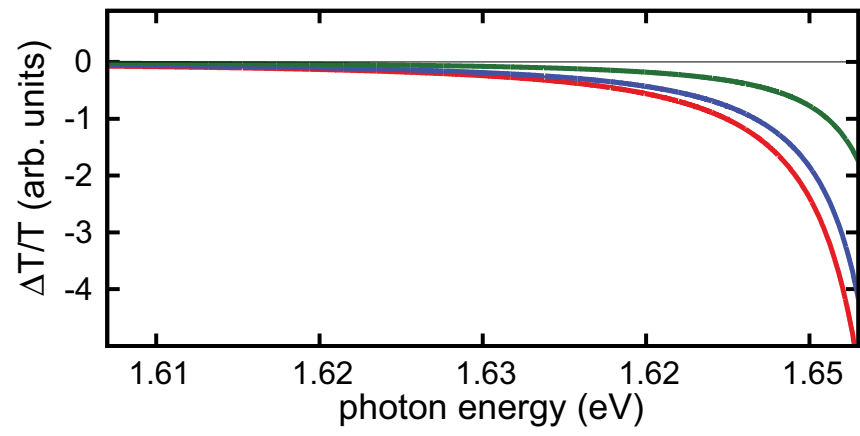

FIG. 4. Calculated differential transmission spectra for $\tau_{d}>0$, namely, $\tau_{d}=1.8 \mathrm{ps}$ (red), $\tau_{d}=4.4 \mathrm{ps}$ (blue), and $\tau_{d}=13.1 \mathrm{ps}$ (green). We use a lifetime of $T_{1}=10 \mathrm{ps}$.

is considerably slowed down due to the exciton-polariton dispersion in the semiconductor. The pump pulse impinges at time $t_{\text {pump }}$ and creates (via two-photon absorption) hot carriers high up in the bands which subsequently relax via scattering processes. Therefore, the hot carrier density $n_{\text {pump }}^{(2)}(t)$ can be described by the time dependence $\Theta\left(t-t_{\text {pump }}\right) \exp \left(-\frac{t-t_{\text {pump }}}{T_{1}}\right)$, where $T_{1}$ is the relaxation time. In order to more realistically model the generation process, the $\Theta$-step function is smoothed by a curve $\frac{1}{2}\left[1+\tanh \left(\frac{t-t_{\text {pump }}}{\Delta t}\right)\right]$, where $\Delta t$ is on the order of $100 \mathrm{fs}$. The carriers together with the probe polarization lead to excitation-induced dephasing [17] in $\delta p^{(3)}(t)$, where $\alpha$ is the strength of the nonlinear process, i.e., the change of the dephasing rate with density. The equations can be solved numerically to obtain the differential transmission $\delta T(\omega) \propto-\operatorname{Im}\left(\frac{\delta P^{(3)}(\omega)}{E_{\text {probe }}(\omega)}\right)$.

As the pump spectrum is centered sufficiently below the LP resonance, we assume a dispersion-free propagation through the sample. It is, however, important to take the frequency dependence of the light velocity for the probe pulse into account. To allow for the fact that the light close to the LP resonance slows down significantly, the probe pulse is numerically propagated using the finite-difference time-domain (FDTD) method based on the auxiliary differential equation technique $[18,19]$. With this method we first determine the probe field $E_{\text {probe }}(t)$ at the end of the $208-\mu \mathrm{m}$ sample. Then we include this propagated field into the $\chi^{(3)}$ equations. Even though this neglects the interaction throughout the full crystal, this simple model is able to qualitatively reproduce the most important features of the measurement. For positive $\tau_{d}>0$ we recover the induced absorption for higher energies, cf. Fig. 4, for results with $T_{1}=10 \mathrm{ps}$. The negative sign suggests excitationinduced dephasing as opposed to Pauli blocking, which would exhibit an opposite sign in the transient optical response. The most pronounced dip is found for the smallest delay. This behavior is expected and its dynamics is governed mainly by the relaxation time $T_{1}$, i.e., the greater $\left|\tau_{d}\right|$, the smaller the induced absorption since less hot carriers are available, thus implying a smaller excitation-induced dephasing.

More interesting is the case $\tau_{d}<0$ where an intricate interplay between frequency-dependent delay times determines the shape of the differential transmission. First of all, the oscillation period is dependent on $\left|\tau_{d}\right|$ : the greater $\left|\tau_{d}\right|$ the faster the oscillations. Even though this is expected because 


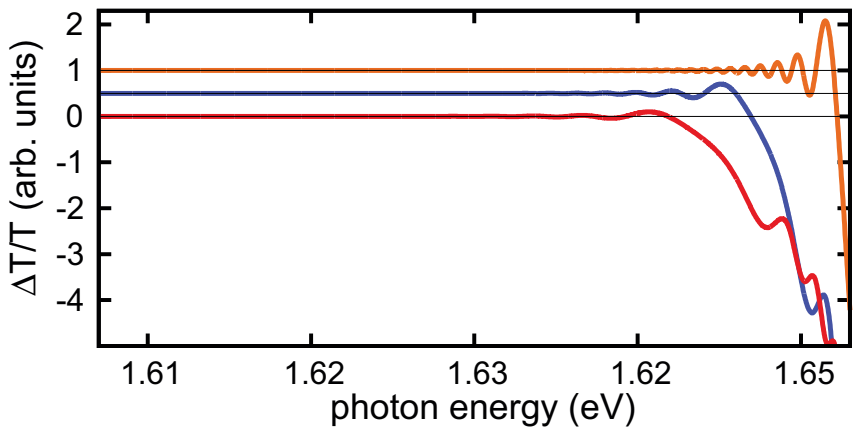

FIG. 5. Calculated differential transmission spectra for $\tau_{d}<0$, namely, $\tau_{d}=-6.2 \mathrm{ps}$ (orange), $\tau_{d}=-2.2 \mathrm{ps}$ (blue), and $\tau_{d}=$ $-1.0 \mathrm{ps}$ (red). The different lines are shifted vertically for clarity.

the period should be $h /\left|\tau_{d}\right|$ for the case of a perturbed free induction decay [14], the measured and calculated oscillation periods are substantially larger.

Another very important feature of Fig. 5 is that the differential transmission shows a change from oscillating behavior to induced absorption. As in the experiment we find that this crossover shifts to lower energies if $\left|\tau_{d}\right|$ is decreased.

In order to comprehend the dependence of the tipping point on $\tau_{d}$, it is helpful to more carefully look at $E_{\text {probe }}(t)$. Figure 6 shows the frequency dependence of the traveling time of the probe pulse at the end of the sample, which has been obtained by multiplying the probe pulse with a Gaussian filter in the frequency domain. The solid line (guide to the eye) indicates that the light slows down considerably when approaching the LP resonance. The dashed line represents the propagation time for the pump pulse in this model. As it is centered far below the LP resonance, it travels through the sample with constant speed. Only via two-photon absorption and scattering processes is the density $n_{\text {pump }}^{(2)}$ created. Thus, one can assume the same timing for the excitation, regardless of the optical frequency within the pump pulse. The intersection point of the solid and dashed curve marks the tipping point in the differential spectra. Intuitively, the spectral position of the tipping point is determined by the largest photon energy where the propagating polariton is surpassed by the excitation front at a given nominal delay time. More specifically, a nominal delay $\tau_{d}<0$ at the front surface of the sample might

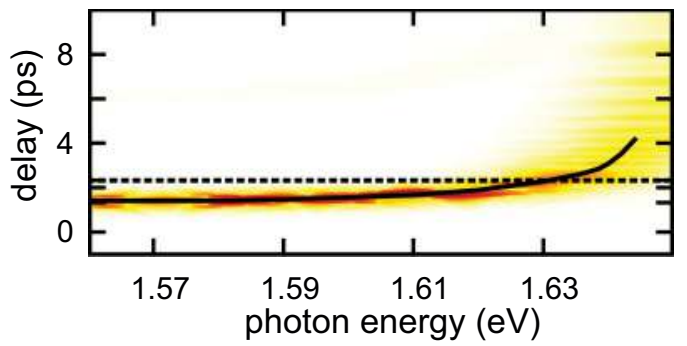

FIG. 6. Frequency dependence of the arrival time of probe light at the rear end of the sample. The pump pulse arrives at the front surface at a nominal time of $\tau_{d}=-1 \mathrm{ps}$ measured with respect to the probe. As $\tau_{d}<0$ some frequency components of the probe (solid) are surpassed by the pump pulse which propagates at a constant velocity (dashed). The solid and dashed lines are a guide to the eye. effectively reverse sign after some propagation distance into the material, at least for certain photon energies. For the example in Fig. $6, \tau_{d}=-1$ ps (measured at the front surface of the sample) has been used. Note that this catch-up effect occurs only for negative delay $\tau_{d}<0$. For $\tau_{d}>0$ in front of the sample a positive delay holds true during the entire propagation through the material. It is, however, true that also in the latter case the time delay between pump and probe pulses becomes larger. In conclusion, for both cases one has to consider a frequency-dependent $\tau_{d}(\omega)$ in the sample rather than the fixed $\tau_{d}$ of the fields impinging on the front surface. With this in mind, also the oscillation periods in Fig. 5, which are significantly larger than naively expected, can be explained.

\section{EXPERIMENTAL RESULTS FOR STRONG EXCITATION}

We now come back to the experimental results and analyze the dependence of the differential transmission spectra on the excitation density. Figure 7(a) shows the results for a fixed delay time of $\tau_{d}=1.3 \mathrm{ps}$. For elevated excitation densities we find very large transmission changes up to a practically complete suppression of the optical transmission over an $\sim 20$-meV broad range for $n_{\mathrm{opt}} \approx 1 \times 10^{16} \mathrm{~cm}^{-3}$. However, this effect is not a purely electronic effect. Increasing the pump power and excitation densities also leads to an increase lattice temperature which, consequently, redshifts the excitonic resonance. Consequently, persistent effects related to thermal heating increasingly influence the transmission of the sample. To differentiate between the electronic and thermal signal contributions, we also analyze the absolute transmitted probe spectrum for the highest excitation density. Figure 7(b) shows these spectra for an irradiance of $200 \mu \mathrm{J} / \mathrm{cm}^{2}$ (corresponding to $n_{\mathrm{opt}}=1 \times 10^{16} \mathrm{~cm}^{-3}$ ) as transmitted through the unexcited (black) and photoexcited (red, delay time $\tau_{d}=-100 \mathrm{ps}$ )
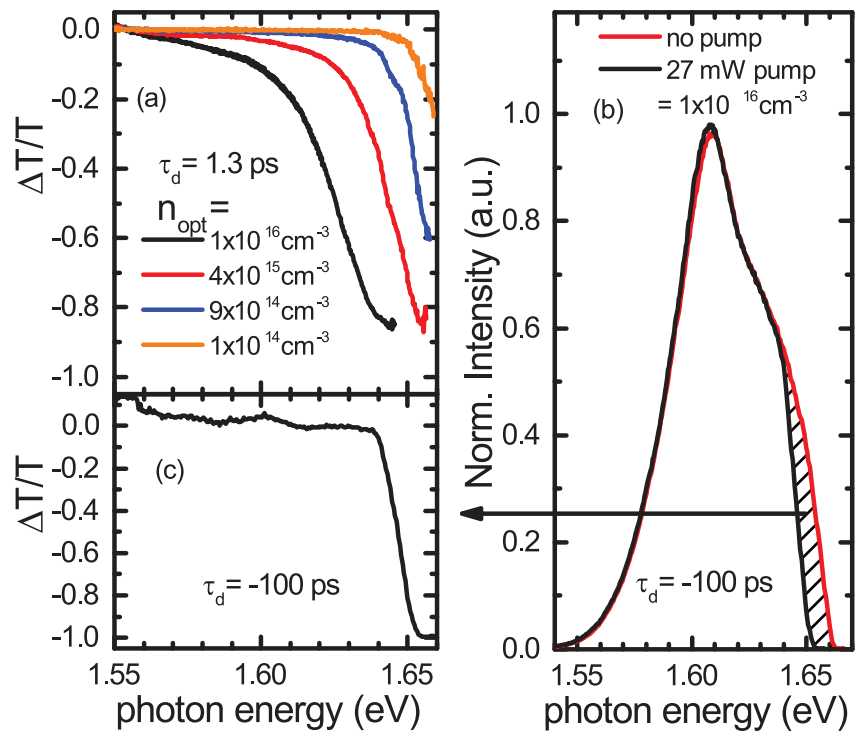

FIG. 7. (a) Differential transmission spectra for various excitation densities and a fixed delay of $\tau_{d}=1.3 \mathrm{ps}$. (b) Normalized transmitted probe spectra taken without (black) and with (red, delay time $\tau_{d}=-100 \mathrm{ps}$ ) excitation of $200 \mu \mathrm{J} / \mathrm{cm}^{2}$ irradiance. (c) Thermal contribution to the differential transmission calculated from the difference of the transmitted spectra in panel (b). 
sample. By analyzing the difference between the two spectra, shown as a hatched gray area, it is now possible to extract the thermal contribution to the differential transmission. The result of this calculation is depicted in panel (c) and shows that even for the present moderate excitation densities, band-gap shifts of $\sim 10 \mathrm{meV}$ and corresponding transmission changes are observed. This finding is in part related to the poor thermal contact in the flow cryostat of our setup.

\section{SUMMARY}

In conclusion, we have investigated the dynamical nonlinear optical response of the lower exciton-polariton branch in high-quality, $\sim 200-\mu$ m-thick $\mathrm{Cd}_{0.88} \mathrm{Zn}_{0.12}$ Te crystals. While the signals at positive delay times are dominated by excitationinduced dephasing, the signatures for nominally negative delay times are more complex. By comparison with model calculations, we identify contributions related to the perturbed free induction decay of the exciton resonance. In addition, excitation-induced dephasing plays an important role, especially for slowly propagating probe components which effectively get surpassed by the much faster excitation front induced by two-photon absorption of the off-resonant pump. Future simulations will focus on a refined analysis of this intricate interaction in optically thick samples.

\section{ACKNOWLEDGMENTS}

This work has been supported by the Sonderforschungsbereich SFB TRR 142 of the Deutsche Forschungsgemeinschaft (DFG).
[1] J. J. Hopfield, Phys. Rev. 112, 1555 (1958).

[2] S. I. Pekar, Zh. Eksp. Teor. Fiz. 33, 1022 (1957) [Sov. Phys. JETP 6, 785 (1958)].

[3] R. G. Ulbrich and G. W. Fehrenbach, Phys. Rev. Lett. 43, 963 (1979).

[4] Y. Segawa, Y. Aoyagi, and S. Namba, Solid State Commun. 32, 229 (1979).

[5] T. Itoh, P. Lavallard, J. Reydellet, and C. Benoit àla Guillaume, Solid State Commun. 37, 925 (1981).

[6] N. A. Vidmont, A. A. Maksimov, and I. I. Tartakovskii, Pis'ma Zh. Eksp. Teor. Fiz. 37, 578 (1983) [JETP Lett. 37, 689 (1983)].

[7] T. V. Shubina, M. M. Glazov, A. A. Toropov, N. A. Gippius, A. Vasson, J. Leymarie, A. Kavokin, A. Usui, J. P. Bergman, G. Pozina, and B. Monemar, Phys. Rev. Lett. 100, 087402 (2008).

[8] T. Godde, I. A. Akimov, D. R. Yakovlev, H. Mariette, and M. Bayer, Phys. Rev. B 82, 115332 (2010).

[9] S. L. Chen, W. M. Chen, and I. A. Buyanova, Phys. Rev. B 83, 245212 (2011).

[10] A. C. Schaefer and D. G. Steel, Phys. Rev. Lett. 79, 4870 (1997).
[11] G. Göger, M. Betz, A. Leitenstorfer, M. Bichler, W. Wegscheider, and G. Abstreiter, Phys. Rev. Lett. 84, 5812 (2000).

[12] E. W. Van Stryland, H. Vanherzeele, M. A. Woodall, M. J. Soileau, and Arthur L. Smirl, Opt. Eng. 24, 244613 (1985).

[13] M. Joffre, D. Hulin, A. Migus, A. Antonetti, C. Benoit àla Guillaume, N. Peyghambarian, M. Lindberg, and S. W. Koch, Opt. Lett. 13, 276 (1988).

[14] M. Lindberg and S. W. Koch, J. Opt. Soc. Am. B 5, 139 (1988).

[15] T. Godde, M. M. Glazov, I. A. Akimov, D. R. Yakovlev, H. Mariette, and M. Bayer, Phys. Rev. B 88, 155203 (2013).

[16] T. Meier, P. Thomas, and S. W. Koch, Coherent Semiconductor Optics: From Basic Concepts to Nanostructure Applications (Springer, New York, 2007).

[17] H. Wang, K. Ferrio, D. G. Steel, Y. Z. Hu, R. Binder, and S. W. Koch, Phys. Rev. Lett. 71, 1261 (1993).

[18] M. Okoniewski, IEEE Microw. Guided Wave Lett. 7, 121 (1997).

[19] A. Taflove and S. C. Hagness, Computational Electrodynamics: The Finite-Difference Time-Domain Method, 3rd ed. (Artech House, Boston, MA, 2005). 